\title{
Primary Jejunal Adenocarcinoma Presenting as Bilateral Ovarian Metastasis
}

\author{
Emmanuel Ofori ${ }^{\mathrm{a}}$, Daryl Ramai ${ }^{\mathrm{a}}$, b, c, Charilaos Papafragkakis ${ }^{\mathrm{a}}$, Kinesh Changela ${ }^{\mathrm{a}}$, \\ Mahesh Krishnaiah $^{\mathrm{a}}$
}

\begin{abstract}
Small intestinal tumors are rare with adenocarcinoma of the small intestine accounting for less than $2 \%$ of all gastrointestinal cancers. Primary jejunal adenocarcinoma constitutes a minute portion of small intestine adenocarcinomas. Clinically, this cancer presents at latter stages of its progression, mainly due to vague and non-specific symptoms, and the difficulty encountered in accessing the jejunum on upper endoscopy. Diagnosis of jejunal adenocarcinoma is usually inconclusive with the use of computed tomography (CT) scan, small bowel series, or upper endoscopy. Laparoscopy followed by frozen section biopsy provides a definitive diagnosis. In the past decade, balloon-assisted enteroscopy (BAE) and capsule endoscopy have become popular as useful modalities for diagnosing small bowel diseases. Wide excisional jejunectomy is the only treatment option with an estimated 5-year survival of $40-65 \%$. Physicians are advised to suspect jejunal adenocarcinoma as a differential diagnosis in patients who present with nonspecific symptoms of abdominal pain, nausea, vomiting, weight loss, anemia, gastrointestinal bleeding or signs of small bowel obstruction. We present a rare case of a 37-year-old woman with suspected bilateral ovarian masses, which was immunohistochemically confirmed as primary jejunal adenocarcinoma with bilateral ovarian metastasis.
\end{abstract}

Keywords: Jejunal adenocarcinoma; Small bowel malignancy; Small bowel tumors; Upper endoscopy

\section{Introduction}

Small intestinal tumors are uncommon worldwide. Specifi-

Manuscript submitted June 21, 2017, accepted September 5, 2017

aDepartment of Gastroenterology and Hepatology, The Brooklyn Hospital Center, Academic Affiliate of The Icahn School of Medicine at Mount Sinai, Clinical Affiliate of The Mount Sinai Hospital, 121 Dekalb Avenue, Brooklyn, NY 11201, USA

bDepartment of Anatomical Sciences, St George's University School of Medicine, True Blue, Grenada, WI

${ }^{\mathrm{c} C}$ Corresponding Author: Daryl Ramai, Department of Anatomical Sciences, St George's University School of Medicine, True Blue, Grenada, WI.

Email: dramai@sgu.edu

doi: https://doi.org/10.14740/gr879w cally, adenocarcinoma of the small intestines is a very rare clinical entity. It accounts for $1-2 \%$ of all gastrointestinal malignancies and about $25-40 \%$ of malignant carcinomas of the small intestines [1]. A large proportion of small intestine malignancy is duodenal adenocarcinoma with primary jejunal adenocarcinoma rarely encountered [2]. Small intestine adenocarcinoma is challenging to diagnose preoperatively mainly due to its non-specific clinical presentation and difficult endoscopic access to the duodenum and jejunum. Additionally, patients present with vague symptoms of abdominal pain, nausea, vomiting, anemia, bleeding and signs of obstruction [3]. As a result, the diagnosis of small intestinal malignancy is usually delayed, missed, or only detected at advanced stages [2]. Herein, we describe a rare case of a 37-year-old woman with suspected bilateral ovarian malignancy, which proved to be a primary jejunal adenocarcinoma with ovarian metastasis. We discuss the salient clinical and pathophysiologic features of this malignancy.

\section{Case Report}

A 37-year-old female with a past medical history of bilateral ovarian tumors status post total abdominal hysterectomy with bilateral salpingo-oophorectomy 1 month prior, presented with abdominal pain. Her abdominal pain was dull, located in the right lower quadrant and epigastric regions of the abdomen, and associated with nausea and non-bloody bilious vomiting. She had regular bowel movements and passed flatus. The patient denied fever, chills, hematochezia or melena. Physical examination noted a soft, non-tender and non-distended abdomen. Laboratory findings showed white cell count of 6,700/ $\mathrm{mm}^{3}$, hemoglobin of $11.5 \mathrm{~g} / \mathrm{dL}$, and normal lactic acid and lipase levels.

Abdominal computed tomography (CT) scan showed mildly dilated loops of small intestine in the left upper quadrant, measuring $3.6 \mathrm{~cm}$ in diameter, with a distal mass-like transition (Fig. 1). Push enteroscopy with a pediatric colonoscope revealed an almost obstructing jejunal mass about 60 $\mathrm{cm}$ distally to the duodenal bulb (Fig. 2). Colonoscopy was unremarkable. Biopsy of the jejunal mass showed an invasive moderately differentiated adenocarcinoma of the small intestine (Fig. 3). The patient underwent laparoscopic small bowel resection with primary anastomosis and omental biopsy. Final pathology report revealed a moderately differentiated, infiltrat- 


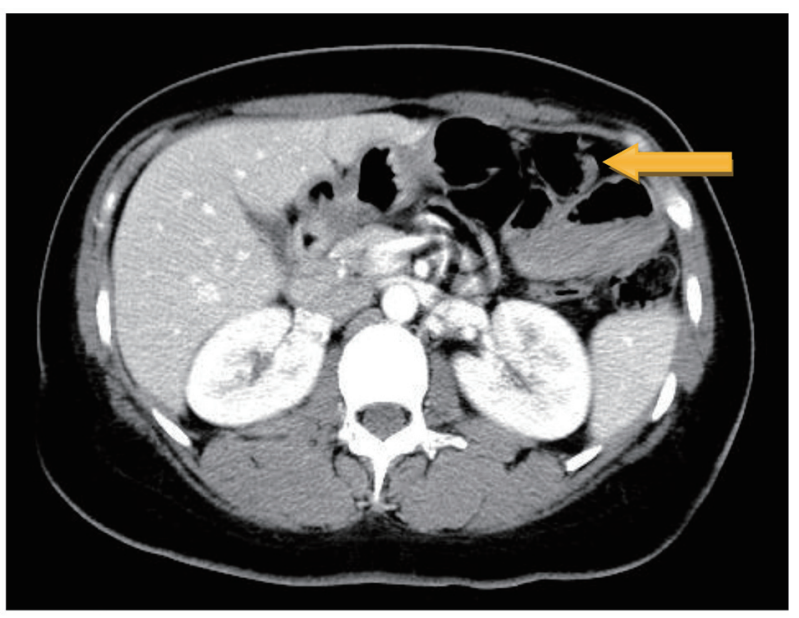

Figure 1. CT scan of abdomen shows dilated loop of small intestine in the left upper quadrant.

ing adenocarcinoma, invading the perijejunal adipose tissue with lymph node involvement.

Immunohistochemical staining revealed that the adenocarcinoma was positive for CDX-2 and CK7, and negative for chromogranin, CK20, and synaptophysin. Histopathological examination of the resected ovarian specimen was CDX2 positive, cytokeratin 7 positive, cytokeratin 20 negative, cytokeratin 5/6 negative, CA-125 negative, mammoglobin negative, estrogen receptor negative, WT-1 negative, napsin negative, and TTF-1 negative. These cytomorphology and immunophenotypical findings strongly supported the diagnosis of primary gastrointestinal adenocarcinoma.

\section{Discussion}

Small intestinal tumors are uncommon, making up about 2.3\% of all gastrointestinal malignancies in the United States [4]. Of these small intestinal malignancies, adenocarcinoma accounts for about $30-40 \%$ with the highest incidence found within the duodenum $[2,4]$. The mean age at diagnosis is generally in the fifth decade of life with a male predominance $[5,6]$. The

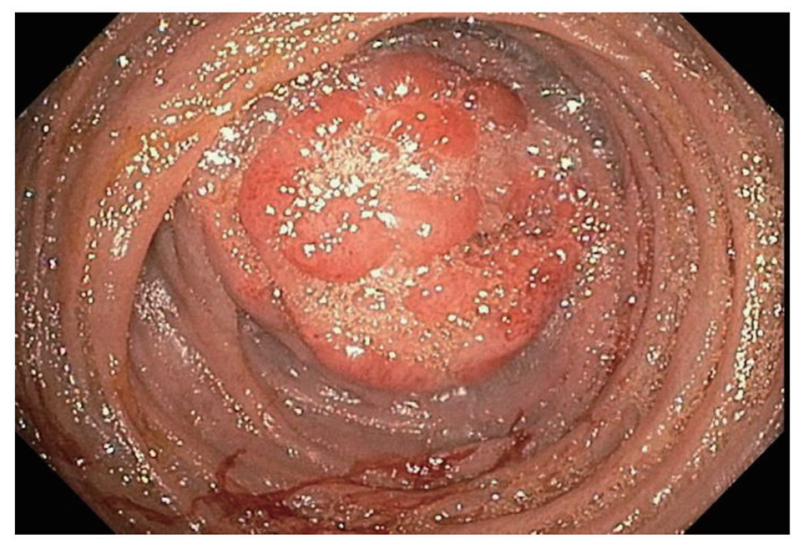

Figure 2. Near obstructing jejunal mass about $60 \mathrm{~cm}$ distally to the duodenal bulb.

clinical presentation of small bowel adenocarcinoma is nonspecific and includes abdominal pain (50-75\%), nausea/vomiting (33-72.5\%), weight loss (38-52.5\%), intestinal obstruction (31.3-44\%), and gastrointestinal bleeding (23-33\%) [6]. Similarly, primary jejunal adenocarcinoma presents with vague symptoms of abdominal pain, nausea and vomiting, anemia, bleeding, weight loss and signs of obstruction [7].

Small intestine adenocarcinoma is rare due to a culmination of various hypothesized explanations such as low bacterial content of the small intestines, presence of enzyme benzopyrene hydroxylase, rapid turnover of epithelial cells, protective lymphoid tissue, and the presence of bile acids $[8,9]$. The fast passage of food through the small intestine provides low mucosal contact time, thereby reducing mucosal injury [4]. Gill et al also hypothesized that the increased levels of IgA expression in the small intestine may serve as mucosal protection against malignancy [10].

In addition to the rarity of jejunal adenocarcinomas, they are usually difficult to diagnose preoperatively. The location of jejunal adenocarcinoma in the small intestine poses difficulty with endoscopic access. As a result, the diagnosis of jejunal adenocarcinoma and other small intestine malignancies is usually delayed, missed, or only detected at advanced stages with poor prognosis [11]. Diagnostic modalities available for de-
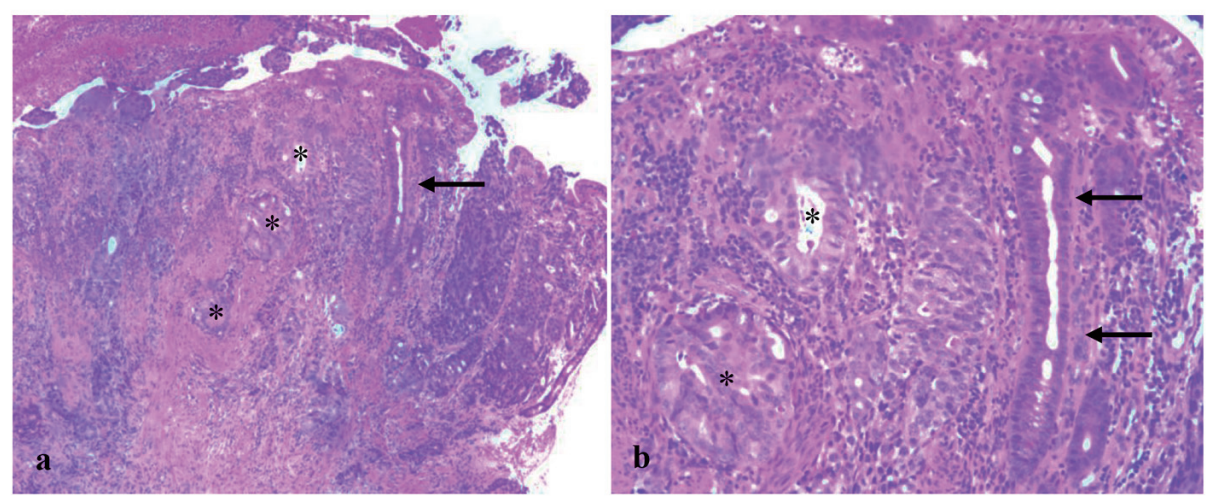

Figure 3. Micrographic examination reveals benign jejunal glands (arrow) and metastatic adenocarcinoma (asterisks) in the lamina propria (a: $\times 40 ;$ b: $\times 100)$. 
tecting small bowel tumors are many; however, they lack an established consensus on the sequence or extent of diagnostic workup. Available for preoperative diagnoses are CT scans, small bowel series, enteroclysis, upper endoscopy, wireless video capsule endoscopy, push enteroscopy and double-balloon endoscopy [11-14]. Laparoscopy followed by frozen section biopsy provides a more definitive diagnosis. However, in the past decade, balloon-assisted enteroscopy (BAE) and capsule endoscopy have gained popularity as useful modalities for diagnosing small bowel diseases.

Cancer-directed surgery provides the best 5-year survival for patients with small intestine cancers. Most studies report a 5 -year survival of $15-35 \%$ with improvement to $40-65 \%$ after surgery [15]. Studies have not shown chemotherapy to provide any significant survival benefit [16].

\section{Conclusion}

Jejunal adenocarcinoma is rare with very dire prognosis. While the clinical outcome of jejunal adenocarcinoma remains grim, it is important to remember that early cancer detection coupled with cancer-directed surgery provides a favorable survival benefit. Physicians should therefore have a high index of suspicion for jejunal adenocarcinoma in patients with vague symptoms of abdominal pain, nausea, vomiting, gastrointestinal bleeding, weight loss, and signs of obstruction. Our case demonstrates the unusual presentation of primary jejunal adenocarcinoma presenting as bilateral ovarian malignancy.

\section{Conflict of Interest}

The authors declare that they have no conflict of interest.

\section{Human/Animal Rights}

All procedures followed were in accordance with the ethical standards of the responsible committee on human experimentation (institutional and national) and with the Helsinki Declaration of 1975, as revised in 2008 [5].

\section{Consent}

Informed consent was obtained from all patients to be included in the study.

\section{References}

1. Weiss NS, Yang CP. Incidence of histologic types of cancer of the small intestine. J Natl Cancer Inst. 1987;78(4):653656.
2. Dabaja BS, Suki D, Pro B, Bonnen M, Ajani J. Adenocarcinoma of the small bowel: presentation, prognostic factors, and outcome of 217 patients. Cancer. 2004;101(3):518-526.

3. Lee HJ, Cha JM, Lee JI, Joo KR, Jung SW, Shin HP. A case of jejunal adenocarcinoma diagnosed by preoperative double balloon enteroscopy. Gut Liver. 2009;3(4):311314.

4. Pan SY, Morrison H. Epidemiology of cancer of the small intestine. World J Gastrointest Oncol. 2011;3(3):33-42.

5. Naef M, Buhlmann M, Baer HU. Small bowel tumors: diagnosis, therapy and prognostic factors. Langenbecks Arch Surg. 1999;384(2):176-180.

6. Talamonti MS, Goetz LH, Rao S, Joehl RJ. Primary cancers of the small bowel: analysis of prognostic factors and results of surgical management. Arch Surg. 2002;137(5):564-570; discussion 570-561.

7. Iusco DR, Altilia F, Massa ST, Schirone S, Villani P, Roggia G. Jejunal adenocarcinoma: still an elusive diagnosis. Case report and review of the literature. Chir Ital. 2008;60(3):457-462.

8. Agrawal S, McCarron EC, Gibbs JF, Nava HR, Wilding GE, Rajput A. Surgical management and outcome in primary adenocarcinoma of the small bowel. Ann Surg Oncol. 2007;14(8):2263-2269.

9. Pilleul F, Penigaud M, Milot L, Saurin JC, Chayvialle JA, Valette PJ. Possible small-bowel neoplasms: contrast-enhanced and water-enhanced multidetector CT enteroclysis. Radiology. 2006;241(3):796-801.

10. Abrahams NA, Halverson A, Fazio VW, Rybicki LA, Goldblum JR. Adenocarcinoma of the small bowel: a study of 37 cases with emphasis on histologic prognostic factors. Dis Colon Rectum. 2002;45(11):1496-1502.

11. Lewis BS, Eisen GM, Friedman S. A pooled analysis to evaluate results of capsule endoscopy trials. Endoscopy. 2005;37(10):960-965.

12. Buckley JA, Siegelman SS, Jones B, Fishman EK. The accuracy of CT staging of small bowel adenocarcinoma: CT/pathologic correlation. J Comput Assist Tomogr. 1997;21(6):986-991.

13. Laurent F, Raynaud M, Biset JM, Boisserie-Lacroix M, Grelet P, Drouillard J. Diagnosis and categorization of small bowel neoplasms: role of computed tomography. Gastrointest Radiol. 1991;16(2):115-119.

14. de Leusse A, Vahedi K, Edery J, Tiah D, Fery-Lemonnier E, Cellier C, Bouhnik Y, et al. Capsule endoscopy or push enteroscopy for first-line exploration of obscure gastrointestinal bleeding? Gastroenterology. 2007;132(3):855862; quiz 1164-1165.

15. Gill SS, Heuman DM, Mihas AA. Small intestinal neoplasms. J Clin Gastroenterol. 2001;33(4):267-282.

16. Young JI, Mongoue-Tchokote S, Wieghard N, Mori M, Vaccaro GM, Sheppard BC, Tsikitis VL. Treatment and survival of small-bowel adenocarcinoma in the United States: a comparison with colon cancer. Dis Colon Rectum. 2016;59(4):306-315. 